\title{
Relationship Between Postural Stability and Falls Risk in Elderly People with Lumbar Spondylosis During Local Vibratory Stimulation for Proprioception: A Cross-sectional Study
}

Tadashi Ito ( $\sim$ tadashi.ito.0805@gmail.com )

Aichi Prefectural Mikawa Aoitori Medical and Rehabilitation Center for Developmental Disabilities https://orcid.org/0000-0002-5427-7609

Yoshihito Sakai

National Center for Geriatrics and Gerontology

Reiya Nishio

Nagoya Institute of Technology

Yohei Ito

Nagoya Institute of Technology

Kazunori Yamazaki

Fujita Health University

Yoshifumi Morita

Nagoya Institute of Technology

Research article

Keywords: falls risk, lumbar spondylosis, muscle spindles, postural strategy, proprioceptive

Posted Date: September 8th, 2019

DOI: https://doi.org/10.21203/rs.2.14121/v1

License: (c) (i) This work is licensed under a Creative Commons Attribution 4.0 International License. Read Full License 


\section{Abstract}

Background: The proprioceptive postural strategy for elderly people with lumbar spondylosis who are at risk for falls has not been extensively investigated. Aims : We aimed to investigate the relationship between proprioceptive postural stability and falls risk in elderly people with lumbar spondylosis.

Methods : In this cross-sectional study, centre-of-pressure displacement was determined in elderly people with lumbar spondylosis during upright stance while standing on a Wii Balance Board with their eyes closed (falls risk group, $n=27$; non-fall risk group, $n=88$ ). Vibratory stimulations at $30 \mathrm{~Hz}$ were applied to the lumbar multifidus and gastrocnemius to evaluate the relative contribution of different proprioceptive signals used in postural control (relative proprioceptive weighting ratio [RPW]). Results: Compared with the non-fall risk group, the falls risk group showed a high RPW at $30 \mathrm{~Hz}(P=0.002)$ and high falls score $(P<0.001)$. Logistic regression analysis revealed that RPW at $30 \mathrm{~Hz}$ (odds ratio, 1.2; 95\% confidence interval: 1.044-1.194) was independently associated with falls risk after controlling for confounding factors. Among variables of falls risk, RPW at $30 \mathrm{~Hz}$ was found to be a significant factor $(P<0.001)$. Discussion: The falls risk group of elderly people with lumbar spondylosis was at a higher risk for falls, which was exacerbated by RPW at $30 \mathrm{~Hz}$ depending on the ankle strategy. A decrease in the input of the muscle spindles in the lumbar multifidus could be a possible mechanism underlying the decreased reliance on lumbar muscle proprioception in these individuals.

\section{Introduction}

Lumbar spondylosis symptoms restrict physical function and mobility and increase the risk of falls in elderly people [1]. Moreover, elderly people at risk of falls experience pain and decline in postural strategy, muscle function, and proprioceptive ability [2-4]. Falls are a common event among elderly people with increased morbidity and disability [5]. Almost $30 \%$ of elderly people aged $\geq 65$ years have recently been reported to experience one or more falls annually, resulting in their death or hospitalization [6, 7]. An important aspect of maintaining one's postural stability, and therefore reducing the risk of falls, is that the sensory gating system should be in conformity with balance control to avoid unpleasant responses triggered by external and internal perturbations [8].

Muscle vibration, known to be a strong stimulus for muscle spindles, has been used to evaluate the role of proprioceptive postural strategy $[3,4,9]$. Because of aging, impairments in the proprioceptive system have been observed to be related with balance control deficits $[10,11]$. Investigating the specific role of proprioception under different conditions of stimulation is essential to gain insight into one's falls risk, variability in postural control strategies, and possibility of impaired proprioception. Toosizadeh et al. reported larger hip sway when maintaining balance under vibratory stimulus in the high-falls risk group of elderly people than in the healthy group [12]. Accordingly, balance impairments, such as variability in postural control strategies and possibility of impaired proprioception, may also lead to an increased risk of falls among elderly people with lumbar spondylosis. However, the association between proprioception and falls risk was not evaluated in these previous studies. Thus, this study aimed to investigate the 
relationship between proprioceptive postural stability and falls risk in elderly people with lumbar spondylosis.

\section{Materials And Methods}

\section{Participants}

This cross-sectional study was performed over 3 years and 3 months (from November 2012 to February 2016 ) in tandem with general clinical practice. During the study period, 115 elderly people (age $>65$ years) living in Aichi, Japan, were recruited for the study and included in the falls risk group (27 participants: 16 women, 11 men) and non-fall risk group (88 participants: 34 women, 54 men). The study participants were patients with spinal column stenosis and spondylitis deformans who presented for conservative treatment of their symptoms. These individuals did not require any assistance to maintain a standing posture. Participants with paralysis, astasia, spinal cord tumour, spinal infection, history of spinal surgery or joint replacement, neurological disorders, balance disorders, vestibular function disorders, and cognitive impairment were excluded.

The same criteria of falls risk used in a previous study were used herein [13]. The participants' history of falls within the past year was recorded using a self-report questionnaire described later herein, and a falls questionnaire score $>7$ indicated a high risk of falls.

\section{Measurements}

\section{Postural stability}

Characteristics of postural stability were measured using a Balance Board (Wii, Nintendo Co., Ltd., Kyoto, Japan) $[9,14-16]$. Balance Board data were acquired using a sampling frequency at $100 \mathrm{~Hz}$ and calculated by Matlab (MathWorks, Inc., Natick, MA, USA) using a low-pass filter with a cutoff frequency at $20 \mathrm{~Hz}$. The participants stood barefoot on the Balance Board with their feet together and eyes closed (Figure 1). Muscle vibration was strongly stimulated bilaterally to the gastrocnemius (GS) and lumbar multifidus (LM) while stimulating the muscle spindles. Vibration was used to evaluate the role of proprioception in postural control $[3,4,8,9]$. The center-of-pressure (COP) displacement of each participant was measured in two muscles under two conditions with one frequency of vibratory stimulation of $0.8 \mathrm{~mm}$ of sinusoidal: (1) $30 \mathrm{~Hz}$ on the GS and (2) $30 \mathrm{~Hz}$ on the $L M[3,4,9,16]$. The measurement time was $30 \mathrm{~s}$, which was divided into two intervals of $15 \mathrm{~s}$ each. Vibratory stimulation was applied to the participants during the last $15 \mathrm{~s}$. We labeled the first $15 \mathrm{~s}$ as "Pre" and the last $15 \mathrm{~s}$ as "During." The participants rested on a chair for $60 \mathrm{~s}$ between measurements. To provide additional information regarding proprioceptive dominance, the relative proprioceptive weighting ratio (RPW) was calculated using the following equation: RPW = (Abs GS) / (Abs GS + Abs LM) $\times 100$ [\%], where Abs GS and Abs LM were the absolute values of the mean COP displacement during GS and LM vibrations, respectively $[4,9,17,18]$. The assessment measures were performed by an experienced doctor and a physiotherapist. 


\section{Low back pain assessment}

Pain was assessed using the visual analog scale (VAS) ( 0 as no pain and 10 as maximum pain) and Roland-Morris disability questionnaire (RDQ) [19]. Participants were asked to complete both pain assessments.

\section{Falls assessment}

All participants were asked to complete the falls questionnaire. Results of the falls assessment were evaluated using a simple falls score [13]. The simple falls score was assessed by closed-ended questions (yes or no) about the participants: (1) Do you have a history of falls within the past 12 months? (2) Has your back become bent? (3) Has your walking speed become slower? (4) Do you use a cane? (5) Do you take 5 or more different medications daily? The fall score ranges from 0 to 13 .

\section{Statistical analysis}

Power analysis was conducted using $\mathrm{G} *$ Power to determine the optimal sample size for a statistical power of 0.8 , an alpha value of 0.05 , a large effect size $(d=0.8)$, two-tailed tests, and an allocation ratio of $0.43[6,7]$. Data in the falls risk group and non-fall risk group were compared using the independent ttest. Stepwise logistic regression analysis was used to assess the relationship between participants' falls risk and RPW. Effect sizes were calculated using $r$ and Cramer's $V$ test (sex). Effect sizes were considered small if $r>0.1$ or -0.1 , moderate if $r<0.3$ or -0.3 , and large if $r<0.5$ or -0.5 . RPW was used as an independent variable and adjusted for age and sex in the stepwise logistic regression analysis. All data were analysed using SPSS for Windows version 19.0 (SPSS Inc., Chicago, IL, USA).

\section{Results}

Power analysis for the sample size revealed that the optimal sample size for this study was 62 participants (non-fall risk group $=43$; fall risk group $=19$ ). Table 1 summarizes the baseline characteristics of study participants and the comparison between the two groups. The falls risk group showed a higher RPW at $30 \mathrm{~Hz}(P=0.002$; Table 2$)$ and higher falls score $(P<0.001$; Table 3$)$ than the non-fall risk group. However, the VAS and RDQ scores were not significantly different between both groups (Table 3). Table 4 presents the factors associated with falls risk in the stepwise logistic regression analysis. Multivariate logistic regression analyses of falls risk after adjustment for age and sex indicated that RPW at $30 \mathrm{~Hz}$ was a significant factor (odds ratio, 1.2; 95\% confidence interval: 1.044-1.194). Age and sex were not significantly different between both groups. Therefore, RPW at $30 \mathrm{~Hz}$ was found to be a significant factor among variables of falls risk.

\section{Discussion}

Our main finding was that the falls risk group decreased their reliance on proprioceptive signals (muscle spindles) from the LM during standing. This reduced LM strategy appears to lead to decreased postural 
stability in proprioceptive inputs from muscle spindles. A previous study has reported reliance on the ankle strategy for postural control during vibratory stimulus in elderly people [2]. On one hand, a smaller influence of muscle tendon vibration on the perception of the dynamic position of the ankle joint has been reported in an elderly group than in a young healthy group [20]. On the other hand, adopting this ankle strategy induced postural instability in the falls risk group [2-4, 21, 22]. The higher RPW values in the falls risk group than in the non-fall risk group during vibratory stimulus at $30 \mathrm{~Hz}$ indicate that the falls risk group relied less on the proprioceptive inputs of the lumbar muscles. This may be because the falls risk group tended to implement the ankle strategy in response to proprioceptive signals (muscle spindles) to maintain balance. Additionally, this result conflicts with that of Toosizadeh et al. who reported that the high falls risk groups showed larger hip sway in the eyes-closed condition than the healthy non-fall risk groups [12]. A reduced multi-segmental strategy seems to lead to decreased postural robustness during vibratory stimulus at $30 \mathrm{~Hz}$ in the falls risk group with lumbar spondylosis [3, 14, 23, 24]. Trunk proprioceptive deficits of muscle spindles have been suggested as a possible reason why the falls risk group could not switch to a more appropriate postural strategy under the condition $[21,22,25,26]$. These findings suggest that there might be differences in strategy selection in elderly people with lumbar spondylosis and a high risk of falls, based on proprioceptive processing from muscle spindles.

Logistic regression analysis showed that the risk of falls increased with an increase of RPW at $30 \mathrm{~Hz}$. This GS strategy in proprioceptive inputs from muscle spindles could lead to less stable postures when the falls score increases. Moreover, the higher RPW values in the falls risk group than in the non-fall risk group when standing on a Balance Board indicate that the falls risk group relied less on lumbar muscle proprioceptive inputs independent of the postural condition. Among the risk factors for falls, the postural strategy with an RPW at $30 \mathrm{~Hz}$ is considered an important risk factor. Additionally, trunk proprioceptive deficits have been attributed to reduced lumbosacral proprioception, which might be a causative factor in elderly people's postural sway during instability $[27,28,29]$. Thus, these results showed that fallers used the ankle strategy more to maintain an upright position, whereas non-fallers predominantly used both the ankle and hip strategies during vibratory stimulation of muscle spindles. Moreover, in this study, a high risk of falls may be likely ruled as a causative factor for the reduction in the variability of the proprioceptive postural strategy of muscle spindles of the trunk because of significant differences in the RPW $30 \mathrm{~Hz}$ between the falls risk and non-fall risk groups. Therefore, these elderly people with lumbar spondylosis may be possibly at a higher risk of falling in the future due to impaired proprioception of the lumbosacral area in muscle spindles.

Despite our notable finding, the results are limited by the cross-sectional design of this study. Only elderly people with lumbar spondylosis were evaluated. In addition to the falls risk, other factors might influence the normal variability of postural control, e.g., fear of falling [30] and exacerbation of low back pain [18]. Further research will be needed to determine whether the risk of falls in reduced trunk proprioceptive input is caused by muscle spindles or by other characteristics of the participants.

\section{Conclusions}


In conclusion, the results of this study underscore the hypothesis that LM proprioceptive impairment from the muscle spindles is associated with a risk of falls in elderly people with lumbar spondylosis. Elderly people with lumbar spondylosis who are at risk for falls exhibit reduced variability in proprioceptive postural strategies for the trunk due to the decreased proprioceptive weighting capacity of muscle spindles. A decrease in the input of the muscle spindles in the LM could be a possible mechanism underlying the decreased reliance on lumbar muscle proprioception in these individuals. An objective assessment of the proprioceptive postural control strategy might be important to identify the limitations or function of elderly people with lumbar spondylosis who are at risk of falls. Future longitudinal research is required to validate these results.

\section{Declarations}

\section{Compliance with Ethical Standards}

Acknowledgments: We thank the staff of the National Center for Geriatric and Gerontology for their help with participant recruitment.

Data statement: All of the relevant data are presented within the manuscript. All data are available from the authors on request.

Contributors: Tadashi Ito and Yoshihito Sakai contributed to the study concept and design; Yoshihito Sakai contributed to study supervision and funding; Reiya Nishio, Yohei Ito, Kazunori Yamazaki, and Yoshifumi Morita contributed to the data analysis, interpretation of the data, and drafting of manuscript; all authors made critical revisions to the manuscript and approved the final version of the manuscript.

Funding: This study was supported in part by a grant from the Geriatrics and Gerontology Research and Development Cost [grant number 26-7].

\section{Disclosure of potential conflicts of interest: None.}

Ethical approval: All procedures performed in studies involving human participants were in accordance with the ethical standards of the institutional and/or national research committee (include name of committee + reference number) and with the 1964 Helsinki declaration and its later amendments or comparable ethical standards. The Ethics Committee of the National Center for Geriatrics and Gerontology approved the study (institutional review board approval number: 586).

Informed consent: Informed consent was obtained from all individual participants included in the study.

\section{References}

1. Kim HJ, Chun HJ, Han CD, et al. (2011) The risk assessment of a fall in patients with lumbar spinal stenosis. Spine (Phila Pa 1976). 36:E588-E592. https://doi.org/10.1097/BRS.0b013e3181f92d8e.

2. Pyykkö I, Jantti P, Aalto H (1990) Postural control in elderly subjects. Age Ageing 19:215-221. 
3. Brumagne S, Cordo P, Verschueren S (2004) Proprioceptive weighting changes in persons with low back pain and elderly persons during upright standing. Neurosci Lett 366:63https://doi.org/10.1016/j.neulet.2004.05.013.

4. Ito T, Sakai Y, Nakamura E, Yamazaki K, Yamada A, Sato N, Morita Y (2015) Relationship between paraspinal muscle cross-sectional area and relative proprioceptive weighting ratio of older persons with lumbar spondylosis. J Phys Ther Sci 27;2247- https://doi.org/10.1589/jpts.27.2247.

5. Thomas DR (2007) Loss of skeletal muscle mass in aging: examining the relationship of starvation, sarcopenia and cachexia. Clin Nutr 26:389- https://doi.org/10.1016/j.clnu.2007.03.008.

6. Liu-Ambrose T, Davis JC, Hsu CL, et al (2015) Action seniors! - secondary falls prevention in community-dwelling senior fallers: study protocol for a randomized controlled trial. Trials 16:144. https://doi: 10.1186/s13063-015-0648-7.

7. Mohler MJ, Wendel CS,Taylor-Piliae RE, Toosizadeh N, Najafi B (2016) Motor performance and physical activity as predictors of prospective falls in community-dwelling older adults by frailty level: application of wearable technology. Gerontology 62:654-664. https://doi.org/10.1159/000445889.

8. Ivanenko YP, Solopova IA, Levik YS (2000) The direction of postural instability affects postural reactions to ankle muscle vibration in humans. Neurosci Lett 292:103-106. https://doi.org/10.1016/s0304-3940(00)01438-5

9. Ito T, Sakai Y, Yamazaki K, Igarashi K, Sato N, Yokoyama K, Morita Y (2017) Proprioceptive change impairs balance control in older patients with low back pain. J Phys Ther Sci 29:1788https://doi.org/10.1589/jpts.29.1788.

10. Horak FB, Shupert CL, Mirka A (1989) Components of postural dyscontrol in the elderly: a review. Neurobiol Aging 10: 727-738.

11. Horak FB (2006) Postural orientation and equilibrium: what do we need to know about neural control of balance to prevent falls? Age Ageing 35:ii7-ii11. https://doi.org/10.1093/ageing/afl077.

12. Toosizadeh N, Ehsani H, Miramonte M, Mohler j (2018) Proprioceptive impairments in high fall risk older adults: the effect of mechanical calf vibration on postural balance. Biomed. Eng Online 17: 51. https://doi.org/10.1186/s12938-018-0482-8.

13. Okochi J, Toba K, Takahashi T, Matsubayashi K, Nishinaga M, Takahashi R, Ohrui T (2006) Simple screening test for risk of falls in the elderly. Geriatr Gerontol Int 28:223-227. https://doi.org/10.1111/j.1447-0594.2006.00352.x.

14. Clark RA, Bryant AL, Pua Y, McCrory P, Bennell K, Hunt M (2010) Validity and reliability of the Nintendo Wii Balance Board for assessment of standing balance, Gait Posture 31:307https://doi.org/10.1016/j.gaitpost.2009.11.012.

15. Young W, Ferguson S, Brault S, Craig C (2011) Assessing and training standing balance in older adults: a novel approach using the 'Nintendo Wii' Balance Board. Gait Posture 33:303https://doi.org/10.1016/j.gaitpost.2010.10.089.

16. Ito T, Sakai Y, Kubo A, Yamazaki K, Ohno Y, Nakamura E, Sato N, Morita (2014) The relationship between physical function and postural sway during local vibratory stimulation of middle-aged 
people in the standing position. J Phys Ther Sci 26:1627-1630.

https://doi.org/10.1589/jpts.26.1627

17. Janssens L, Brumagne S, McConnell AK et al (2013) Proprioceptive changes impair balance control in individuals with chronic obstructive pulmonary disease. PLoS One 8:e57949. https://doi.org/10.1371/journal.pone.0057949.

18. Ito T, Sakai S, Morita Y, Yamazaki K, Igarashi K, Nishio R, Sato N (2018) Proprioceptive weighting ratio for balance control in static standing is reduced in elderly patients with non-specific low back pain. Spine (Phila Pa 1976) 43:1704-1709. https://doi.org/10.1097/BRS.0000000000002817.

19. Roland M, Morris R (1983) A study of the natural history of back pain. Part I: development of a reliable and sensitive measure of disability in low-back pain. Spine 8:141-144. https://doi.org/10.1097/00007632-198303000-00004

20. Verschueren S, Brumagne S, Swinnen S, Cordo P (2002) The effect of aging on dynamic position sense at the ankle. Behav Brain Res 136:593-603.

21. Brumagne S, Janssens L, Janssens E, Goddyn L (2008) Altered postural control in anticipation of postural instability in persons with recurrent low back pain. Gait Posture 28:657-662. https://doi.org/10.1016/j.gaitpost.2008.

22. Claeys K, Brumagne S, Dankaerts W, Kiers H, Janssens L (2011) Decreased variability in postural control strategies in young people with non-specific low back pain is associated with altered proprioceptive reweighting. Eur J Appl Physiol 111:115- https://doi.org/10.1007/s00421-010-1637$x$.

23. Ivanenko YP, Wright WG, Gurfinkel VS, Horak F, Cordo P (2006) Interaction of involuntary postcontraction activity with locomotor movements. Exp Brain Res 169:255-260. https://doi.org/10.1007/s00221-005-0324-3

24. Gilhodes JC, Gurfinkel VS, Roll JP (1992) Role of la muscle spindle afferents in post-contraction and post-vibration motor effect genesis. Neurosci Lett 135:247-251. https://doi.org/10.1016/03043940(92) $90447-f$

25. Eklund G (1972) General features of vibration-induced effects on balance. Ups J Med Sci 77:112124. https://doi.org/10.1517/03009734000000016

26. Quoniam C, Hay L, Roll JP, Harlay F (1995) Age effects on reflex and postural responses to propriomuscular inputs generated by tendon vibration. J Gerontol A Biol Sci Med Sci 50:B155-B165. https://doi.org/10.1093/gerona/50a.3.b155

27. Newcomer KL, Laskowski ER, Yu B, Johnson JC, An KN (2000) Differences in repositioning error among patients with low back pain compared with control subjects. Spine (Phila Pa 1976) 25:24882493. https://doi.org/10.1097/00007632-200010010-00011

28. Brumagne S, Cordo P, Lysens R, Verschueren S, Swinnen S (2000) The role of paraspinal muscle spindles in lumbosacral position sense in individuals with and without low back pain. Spine (Phila Pa 1976) 25:989-994. https://doi.org/10.1097/00007632-200004150-00015 
29. Ito T, Sakai Y, Yamazaki K, Nishio R, Ito Y, Morita Y (2018) Postural strategy in elderly, middle-aged, and young people during local vibratory stimulation for proprioceptive inputs. Geriatrics 3:93. https://doi.org/10.3390/geriatrics3040093.

30. Isableu B, Vuillerme N (2006) Differential integration of kinaesthetic signals to postural control. Exp Brain Res 174;763-768. https://doi.org/10.1007/s00221-006-0630-4.

\section{Tables}

э 1. Demographic characteristics of the participants.

\begin{tabular}{lcccc}
\hline ariable & $\begin{array}{c}\text { Falls risk group (n } \\
\text { = 27) }\end{array}$ & $\begin{array}{c}\text { Non-fall risk group (n } \\
\text { = 88) }\end{array}$ & $\begin{array}{c}\text { P- } \\
\text { value }\end{array}$ & $\begin{array}{c}\text { Effect size } \\
\text { (r or Cramer's V } \\
\text { test) }\end{array}$ \\
\hline э (years) & $77.0(67-84)$ & $74.5(65-85)$ & 0.102 & -0.15 \\
Sex & $11 / 16$ & $54 / 34$ & 0.059 & 0.06 \\
e/female) & & & & \\
\hline ght $(\mathrm{cm})$ & $152.4 \pm 10.6$ & $156.6 \pm 8.1$ & 0.029 & 0.20 \\
ight $(\mathrm{kg})$ & $58.3 \pm 11.1$ & $59.3 \pm 10.9$ & 0.674 & 0.06 \\
\hline $\mathrm{I}\left(\mathrm{kg} / \mathrm{m}^{2}\right)$ & $25.1 \pm 3.9$ & $24.0 \pm 3.2$ & 0.163 & 0.13
\end{tabular}

body mass index; RDQ, Roland-Morris disability questionnaire; SD, standard ttion; VAS, visual analog scale. Data are presented as the mean \pm SD or $\mathrm{n}$. All pis were generated using the independent t-test or chi-square test. 
2. Mean displacements of the proprioceptive weighting ratio during the vibration for both groups.

\begin{tabular}{lcccc}
\hline ariable & Falls risk group $(\mathrm{n}=$ & Non-fall risk group $(\mathrm{n}=$ & P- & Effect size \\
& 27) & 88) & value & $(\mathrm{r})$ \\
\hline at $30 \mathrm{~Hz}$ & $55.6 \pm 9.6$ & $48.8 \pm 7.0$ & 0.002 & 0.50
\end{tabular}

tandard deviation; RPW: proprioceptive weighting ratio. Data is presented as the \pm SD. P-value was generated using the independent t-test.

э 3. Results of questionnaire assessments in both groups.

\begin{tabular}{lcccc}
\hline iable & $\begin{array}{c}\text { Falls risk group }(\mathrm{n}= \\
\text { 27) }\end{array}$ & Non-fall risk group $(\mathrm{n}=\mathbf{8 8})$ & P-value Effect size (r) \\
\hline score & $5.0(0-8.0)$ & $5.0(0-10.0)$ & 0.532 & -0.06 \\
\hline score & $13.0(2-23)$ & $11.0(0-23)$ & 0.062 & -0.17 \\
ills & $9.0(8-13)$ & $4.0(0-6)$ & 0.001 & -0.75 \\
ore & & & & \\
\hline
\end{tabular}

: Roland-Morris disability questionnaire; VAS: visual analog scale. Data are snted as the median values (minimum and maximum values). The $\mathrm{p}$ values were 'mined using the Mann-Whitney U-test. 
le 4. Factors associated with the falls risk in stepwise logistic regression analysis $(\mathrm{n}=$ ) .

\begin{tabular}{lccc}
\hline Variable & OR & 95\% CI & P-value \\
\hline W at $30 \mathrm{~Hz}$ & 1.2 & $1.044-1.194$ & 0.001 \\
e & 1.1 & $0.997-1.205$ & 0.059 \\
\hline x & 0.6 & $0.215-1.508$ & 0.257 \\
\hline
\end{tabular}

confidence interval; OR: odds ratio; RPW: proprioceptive weighting ratio. Hosmerleshow $\chi^{2}=8.406, \mathrm{p}=0.395$.

\section{Figures}

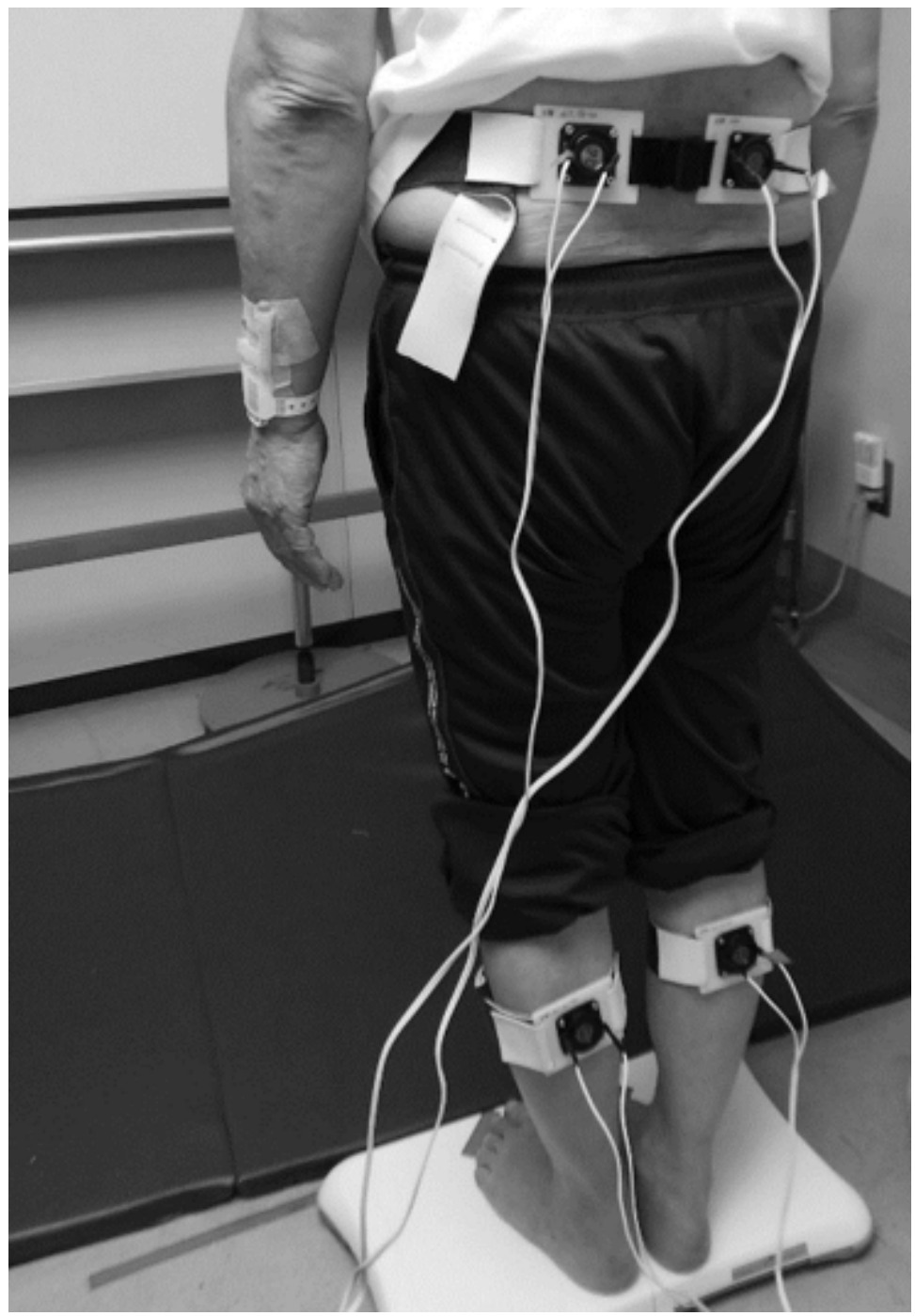

Figure 1 
Experimental setup. Vibration is applied to a participant's gastrocnemius and lumbar multifidus during upright standing while on a Wii Balance Board 